\title{
Correction to: Instability of a Flow System
}

\section{Correction to:}

\section{Chapter 4 in: A. Barletta, Routes to Absolute \\ Instability in Porous Media, https://doi.org/10.1007/978-3-030-06194-4_4}

The original version of Chap. 4 was inadvertently published with errors in several equations and text that under come Sect. 4.4 which conflict with some of the conclusions. These errors have been corrected and the Fig. 4.9 has also been updated with relation to the corrected equation.

The correction chapter and the book have been updated with the change. 